\title{
Interconexión de Mercados Transfronterizos de Electricidad y la Necesidad de Políticas de Coordinación
}

\section{Cross-border interconnection of electrical power markets and the need for policy coordination}

\author{
Fabiola Jara ${ }^{*}$, Jaime Luyo ${ }^{1^{*}}$, Alberto Rios ${ }^{2}$ \\ ${ }^{1}$ Facultad de Ingenia Mecánica, Universidad Nacional de Ingeniería, Av. Tupac Amaru 210, Rimac, Lima, Perú. \\ ${ }^{2}$ Faculty of Sytems, Electronics and Industrial Engineering Universidad Técnica de Ambato, Ambato, Ecuador. \\ Recibido (Received): 01/08/2018 \\ Aceptado (Accepted): 11/12/2018
}

\begin{abstract}
RESUMEN
La interconexión de dos mercados eléctricos necesita nuevas políticas de regulación que la rijan, porque hay diversos factores que se involucran cuando se interconectan dos mercados, ya que las políticas del mercado de electricidad de cada país están diseñadas para su mercado interno, por lo que existe la necesidad de determinar cuál es el mejor mecanismo para la interconexión de ambos mercados, tratando que ambos países compartan los beneficios y los costos. Estudiar el impacto de la participación de grandes centrales hidroeléctricas con embalse y el efecto que tendrán en la complementariedad de los mercados eléctricos transfronterizos; la implementación de RER no convencionales y como afectaran en la integración de los mercados eléctricos transfronterizos, son problemas que no tienen soluciones generales y que deben tratarse para cada caso específico.
\end{abstract}

Palabras clave: (Interconexión, mercado de electricidad, políticas de regulación, simulación, complementariedad)

\section{ABSTRACT}

The interconnection of two power grids creates a need for new regulation policies to govern it, as many factors are involved when two markets become interconnected and the policies of each country are designed only for its internal power market. It is therefore necessary to find the best mechanism for interconnecting both markets, which will also ensure that both countries share the benefits and costs. Issues like the contribution of large hydroelectric plants with reservoirs, the implementation of unconventional renewable energy sources and the effect of both on the complementarity and integration of cross-border power markets; all are problems that have no general solution and have to be solved for each particular case.

Keywords: (Interconnection, electricity market, regulation policies, simulation, complementarity)

\section{INTRODUCTION}

Los sistemas eléctricos desde su creación son operados de forma independiente y diferente en cada país, debido a que las políticas regulatorias son formuladas de acuerdo con el tipo de mercado de electricidad que poseen; así como, de su política económica. Sin embargo, en las últimas décadas algunos países optaron por la interconexión transfronteriza de sus sistemas eléctricos con el objetivo de reducir sus costos de producción, maximizar el aprovechamiento de las fuentes de generación, diversificar su matriz de generación de electricidad y reducir la emisión de los gases de efecto invernadero.

Si bien es cierto la interconexión de sistemas eléctricos, maximiza el beneficio social global de ambos sistemas en su conjunto, se debe tener en cuenta que si se evalúa cada sistema eléctrico de forma independiente resulta una variación de costos de generación y precios de electricidad en ambos lados de la interconexión, produciéndose una distribución desequilibrada en los beneficios. Para llevar a cabo la integración de sistemas eléctricos de diferentes países se deben analizar aspectos importantes como los marcos legales y regulatorios; así como, sus respectivas políticas de interconexión de cada país de forma independiente y recién adoptar la decisión de interconexión.

En los últimos años se interconectaron los sistemas eléctricos de muchos países, llegando a acuerdos aparentemente beneficiosos para ambos sistemas. La interconexión en américa latina que actualmente está implementada es la interconexión transfronteriza Ecuador-Colombia. Esta interconexión se realizó porque

\footnotetext{
* Correspondencia:

E-mail: fabiolajaraca@gmail.com
} 
se estudiaron las diversas políticas de interconexión a fin de aprovechar la complementariedad hídrica, ya que cuando en Ecuador es época de avenida, en Colombia es época de Estiaje, y viceversa.

Asimismo, existen el potencial para la interconexión de muchos países latinoamericanos; sin embargo, los procedimientos de intercambios de energía no están en funcionamiento, ya sea por falta de estudios, o falta de coordinación de mercados. Cada mercado eléctrico nacional tiene características particulares en la compra y venta de energía eléctrica, esto se debe a que a nivel interno cada país implementa políticas regulatorias para garantizar su confiabilidad a corto plazo y autosuficiencia a largo plazo en su capacidad de generación para cubrir el crecimiento de la demanda eléctrica. Sin embargo, estos criterios políticos son formulados con un enfoque nacional y no con una orientación hacia la integración energética regional.

Por lo tanto, existe la necesidad de estudiar el problema de "la distribución de los incrementos y reducciones de los beneficios entre los agentes en mercados eléctricos interconectados", como es el caso de estudio de la interconexión Perú-Chile, con el objetivo de Modelar y simular la integración de los mercados eléctricos transfronterizos entre Perú y Chile. Para proponer soluciones que permitan superar los obstáculos en el ámbito normativo y regulatorio del comercio de electricidad transfronterizo que vincula a ambos mercados eléctricos.

\section{ANTECEDENTES}

La integración de los mercados de energía eléctrica se empezó a desarrollar bajo la tendencia de la liberación económica a nivel mundial, en el año 2000 se establecieron algunos acuerdos en América latina, con el propósito de alcanzar el abastecimiento total de energía eléctrica, debido a que algunos de los países no son autosuficientes y para alcanzar su sostenibilidad energética se realizaron algunas interconexiones transfronterizas [1]; para que dichas interconexiones se incrementen se tienen que superar algunos retos, ya que se concluyó que es muy difícil llegar a acuerdos porque las políticas internas de regulación que rigen las normas de los países obstaculizan el funcionamiento de estos acuerdos, otro de los factores es la diferencia entre los tipos de mercados de electricidad; sin embargo, la interconexión puede servir para mejorar la organización y diseño de dichos mercados de electricidad [2-3].

Otro punto muy importante es determinar la capacidad de generación total que posee un el sistema eléctrico de un país en la hora pico, para así dimensionar la demanda requerida por el país vecino. Este problema es mucho más serio si el flujo de potencia se entrega en una sola dirección, ya que los usuarios finales del país exportador serian perjudicados, pero sí, se mejoraría el bienestar global; y si ambos mercados pretenden maximizar sus ganancias, la interconexión podría ser factible; sin embargo, los países deben elegir un diseño optimo con el fin de que los consumidores del país exportador sean perjudicados lo menos posible [4-5].

Camila Ochoa [6] estudió las interconexiones eléctricas transfronterizas desde el punto de vista técnico-económico, y concluyó que las interconexiones son beneficiosas, pero no se tiene que olvidar el hecho de que las políticas de interconexión deben promover más el acondicionamiento de la oferta en el mercado eléctrico a largo plazo, con el fin de promover el financiamiento y construcción de nuevas centrales eléctricas para que los países con el tiempo sean capaces de autoabastecerse.

En los últimos años se está implementando intensivamente el uso de energías renovables no convencionales, sin embargo, se tiene problema "del mecanismo de capacidad remunerativa" lo que aún se está discutiendo mediante dos alternativas, (I) Estrategias de reserva y (II) pagos por capacidad u opciones de confiabilidad, lo cual permitirá que estas fuentes puedan entrar en la comercialización transfronteriza [7].

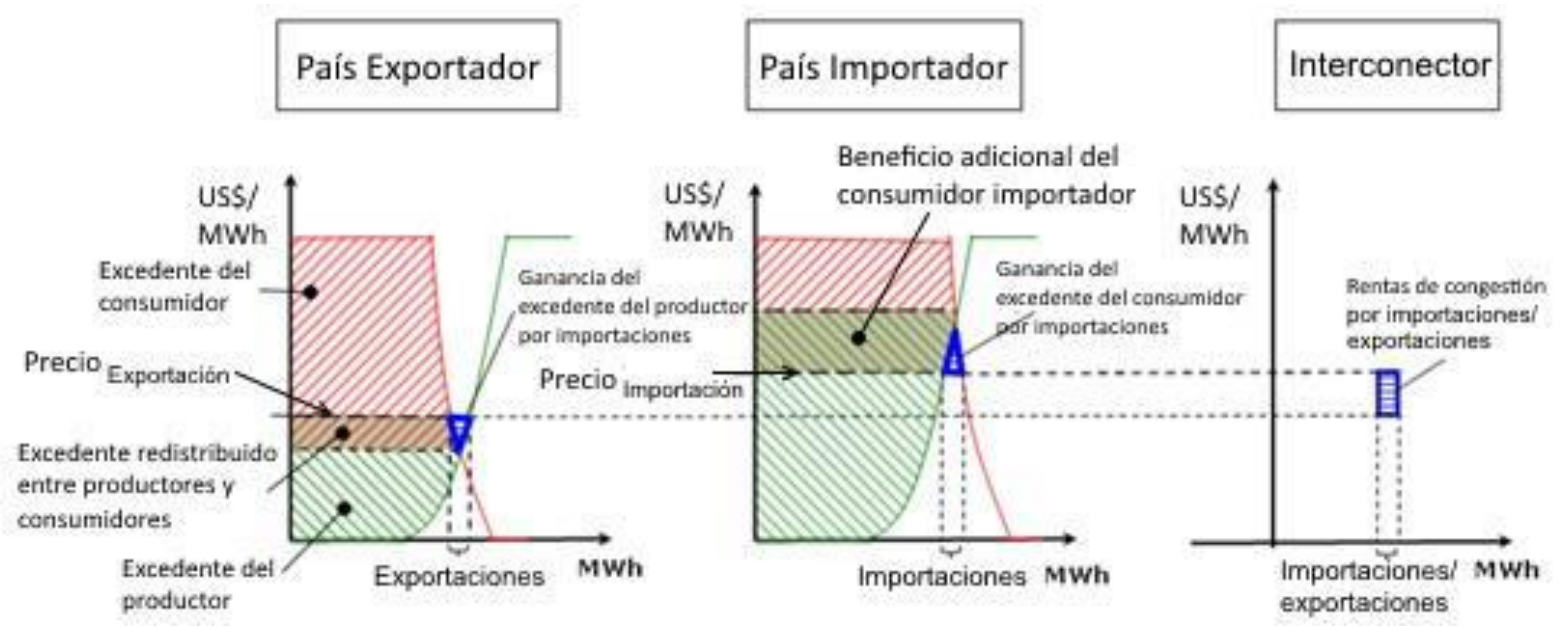

FIGURA 1 Exportación - importación de electricidad entre dos países 
Las interconexiones son en general convenientes, ya que los recursos energéticos que poseen los países no son igualmente distribuidos, además pueden contribuir a reducir los precios de energía e incrementar el suministro en regiones con mayor demanda, aumentando los ingresos del país exportador [8]

Finalmente, en la integración de mercados eléctricos entre países que tengan precios elevados y precios bajos, se espera que traiga beneficios a los consumidores de países con precios elevados, pero esto dependerá del tipo de políticas de regulación que se implementen. La venta de energía desde el país con precios bajos hacia el país que tiene altos precios es conveniente debido a que el país exportador genera ingresos y los consumidores del país importador tendrán electricidad a un precio más económico [9] como se presenta en la Figura 1.

\section{FACTORES INSTITUCIONALES, TÉCNICOS Y ECONÓMICOS}

\subsection{ESTUDIOS DE PARA LA INTERCONEXIÓN PERÚ - CHILE}

Los Estudio de Deloitte/Black \& Veatch [10] y [11] se han enfocado solo en la evaluación de impacto del interconector en los costos de producción en Perú y Chile, debido a las limitaciones de diseño del modelo empleado (Programación Dinámica Dual Estocástica) que es de despacho económico y no es para el estudio de mercados eléctricos competitivo. Además, resulta observable los supuestos como: la eliminación de congestiones en las líneas de transmisión; la configuración "coordinada" de los sistemas SEIN-SINGSIC, entre otros. En el Estudio, para todas las 16 opciones analizadas resultan ahorros (descontando incluso el costo del Interconector) en un rango que, potencialmente pueden multiplicarse los ahorros del caso base en 15 veces.

En el comercio internacional, dentro de la actual corriente neoliberal, hay ganadores y perdedores. En el caso del comercio de electricidad Perú-Chile, según las condiciones técnico-económicas del SEIN-SING-SIC, al Perú le corresponderá jugar de perdedor [12].

\subsection{DESCISIONES DE LA COMUNIDAD ANDINA DE NACIONES SOBRE INTEGRACIÓN ELECTRICA}

J.Luyo [13], señala que en La integración eléctrica regional es un proceso que se ha venido desarrollando en forma lenta, particularmente dentro del ámbito de la Comunidad Andina de Naciones (CAN), reducida con el retiro de Chile y Venezuela, y está tomando un mayor impulso en la última década con la interconexión Ecuador-Colombia y la conexión temporal Perú-Ecuador hacia la conformación del Sistema de Interconexión Eléctrica Andina (SINEA). Después de la adopción de la Decisión 536 de 2002 y que fue suspendida, hubo doi: https://doi.org/10.21754/tecnia.v28i2.590 avances y retrocesos en decisiones posteriores. En la actualidad, hay propuesta una nueva Decisión que establece, entre otros, que:

a) Sólo se exportará excedentes de corto plazo (despacho 24 horas). Sin firmeza. No se requiere pago por capacidad

b) Se introduce el concepto de Mercado del día previo (vinculante) y de los mercados intra diarios.

c) Se distingue los precios del Mercado interno de los precios del resto.

d) Las rentas congestión se asignarán 50\% - 50\% entre los países importador y exportador.

e) Solo configura un mercado de corto plazo entre operadores del mercado. No se requiere contratos comerciales para habilitar las transacciones.

f) No se prevé regulación sobre contratos bilaterales entre agentes.

g) Las transacciones físicas internacionales serán consecuencia del despacho económico de los excedentes de producción.

h) No se exportará subsidios.

i) El pago por el uso de las redes se efectuará a través del concepto de peaje. No se requiere el concepto de país de tránsito para la remuneración de la transmisión.

La propuesta de la CAN de fijar "los precios del mercado interno de los precios del resto" requiere una intervención directa de los operadores en el mercado de electricidad, violando los fundamentos del libre mercado; con la complicación adicional de la distribución de los ingresos por exportación.

Solo tener un mercado de corto plazo en la CAN limitará la posibilidad de optimizar la expansión de generación. Se debe revisar la distribución igualitaria de rentas de congestión y considerar que en el Perú hay subsidio implícito al gas natural.

\section{CONCLUSIONES}

Para analizar adecuadamente las características antes señalados se plantea la necesidad de desarrollar un modelo de expansión de la generación, para ello se puede emplear la teoría de dinámica de sistemas $[14,15]$

A partir de la teoría de dinámica de expansión de los sistemas eléctricos [16], se desarrollará un modelo de Dinámica de Sistemas para la expansión de la generación en un país. El horizonte de simulación de largo plazo, con un paso mensual. Este tiempo es suficiente para observar la expansión en las diferentes tecnologías de generación, y el paso mensual permite observar las variaciones estacionales de la oferta debidas a la hidrología.

Asimismo, se requiere un modelo de despacho económico de corto plazo para determinar el impacto 


\section{F. Jara et al.}

en los costos marginales que afectarán la rentabilidad de la capacidad de interconexión, asimismo se requiere que la interacción entre ambos modelos sea dinámico.

\section{REFERENCIAS}

[1] C. Ochoa, F. J. Carlos 2013 Energy Policy. 61 267-273.

[2] R. Poudined, A. Rubino 2017 Energy Policy.107 96-108.

[3] O. Gore, S. Viljainen 2014 in International Conference on the European Energy Market, Lappeenranta, Finland.

[4] M. Roland, O. Gore 2015 Energy Economics 51 9-20.

[5] C. Ochoa, A. v. Ackere 2015 Energy 80 522-534.

[6] C. Ochoa, A. v. Ackere 2015 Renewable and Sustainable Energy Reviews 50 1108-1124.

[7] R. Meyer, O. Gore, G. Brunekreeft y S. Viljainen 2014 Elforsk rapport.
[8] P. Meisen, 2010 Global Energy Network Institute.

[9] C. Ochoa, O. Gore 2015 Energy Policy 80 122-132.

[10] Deloitte, Black\&Veatch 2015 "Estudio de Análisis para la Planificación"

[11] Deloitte, Black\&Veatch 2015 "Estudio de Análisis Técnico"

[12] J. Luyo 2017 "Interconexión Eléctrica Perú-Chile: Factores institucionales, técnicos y económicos"

[13] J. Luyo 2017 EL Ingeniero. 8032

[14] J. D. Sterman 2000 Business Dinamics: Systems Thinking and Modeling for a Complex World McGraw-Hill USA.

[15] M. Ruth y B. Hannon 2012 Modeling Dynamic Systems 2 Springer.

[16] A. Ford 1997 System Dynamics Review 13 57-85.

Los artículos publicados por TECNIA pueden ser compartidos a través de la licencia Creative Commons: CC BY 4.0 Perú. Permisos lejos de este alcance pueden ser consultados a través del correo revistas@uni.edu.pe 